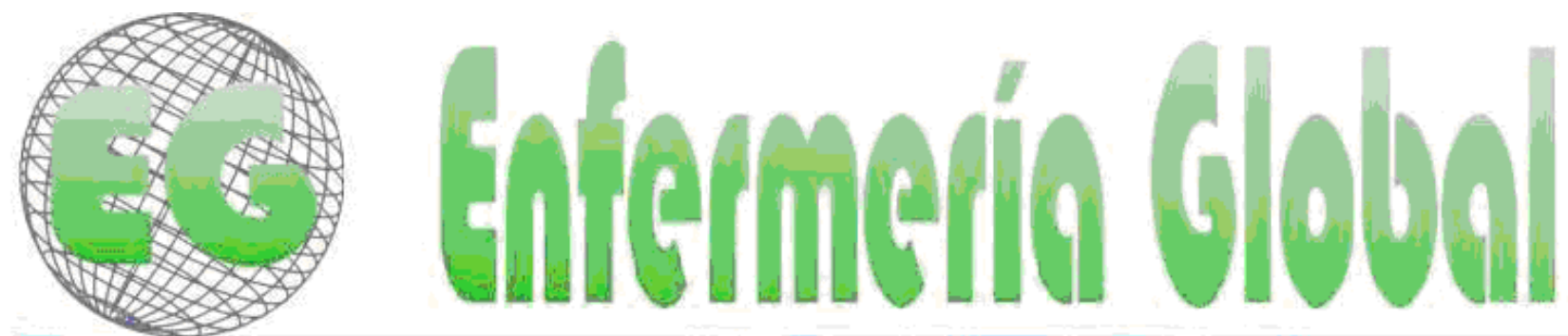

1SSN $1695-6144$

$\mathrm{N}^{\circ} 16$

Revista electrónica cuatrimestral de Enfermería

Junio 2009

www.um.es/eglobal/

ADMINISTRAGIÓN - GESTIÓN - GALIDAD

\title{
PREPARACIÓN DE MATERIALES EN SALAS DE DEPÓSITO INSTRUMENTAL Y DE MATERIAL RESIDUAL: UNA REFLEXIÓN SOBRE ESTA PRÁCTICA
}

PREPARO DE MATERIAIS EM SALAS DE UTILIDADES: UMA REFLEXÃO SOBRE ESTA PRÁTICA

\begin{abstract}
${ }^{*}$ Oliveira, AC., ${ }^{* *}$ Costa, TMPF, ${ }^{* * *}$ Rodrigues Ribeiro, M., ${ }^{* * * *}$ Oliveira de Paula, A., ${ }^{* * * *}$ Azevedo, TC.

*Doutora en Enfermagem. Professora Adjunta do Departamento de Enfermagem Básica da Escola de Enfermagem da UFMG, membro da Comissão de Controle de Infecção Hospitalar do Hospital das Clínicas da UFMG. ***Professora Assistente do Departamento de Enfermagem Básica da Escola de Enfermagem da UFMG, membro da Comissão de Controle de Infecção Hospitalar do Hospital das Clínicas da UFMG. *** Enfermeira. Membro da Comissão de Controle e Infecção Hospitalar do Hospital das Clínicas da UFMG. ****Alunos do Curso de Graduação em Enfermagem da Escola de Enfermagem da UFMG. Voluntários do Projeto de Pesquisa "Reprocessamento de materiais no HC/UFMG" . Brasil.
\end{abstract}

Palabras Clave: equipo reutilizado; infección hospitalaria; prestación de atención de salud; capacitación en servicio

Palavras-chave: reutilização de equipamento; infecção hospitalar; assistência à saúde; capacitação em serviço

\section{RESUMEN}

El objetivo fue describir el perfil de los profesionales que trabajan en el depósito de instrumental y material residual (SDI) de un hospital universitario, en lo concerniente a la formación, supervisión, disponibilidad de rutinas sanitarias, entrenamiento en el trabajo y en preparación de los materiales. Se trató de un estudio descriptivo y de enfoque cuantitativo realizado en el período que va de octubre de 2006 a febrero de 2007. Para recolección de los datos se utilizó un cuestionario semiestructurado. Durante el estudio, 11 unidades fueron analizadas contando con 55 profesionales actuantes en la SU. Se constató un predominio de auxiliares de enfermería $(60,0 \%)$, seguidos por técnicos de enfermería $(36,6 \%)$ y enfermeros $(3,4 \%)$. Principales resultados: diversidad de conductas frente al procedimiento de limpieza y desinfección de los materiales; falta de supervisión directa del enfermero en la SU, de manual de normas y rutinas, y de un programa de educación continuada. Estos resultados ponen de relieve la necesidad de establecer estrategias dirigidas a la prevención y control de los riesgos en lo que atañe a las prácticas de preparación de los materiales en la institución materia de estudio. 


\section{RESUMO}

Objetivou-se descrever o perfil dos profissionais atuantes nas salas de utilidades (SU) de um hospital universitário, quanto à formação, supervisão, disponibilidade de rotinas no setor, treinamentos em serviço e preparo dos materiais. Tratou-se de um estudo descritivo e de abordagem quantitativa realizado no período de outubro de 2006 a fevereiro de 2007. Para a coleta de dados utilizou-se um questionário semi-estruturado. Durante o estudo onze unidades foram avaliadas sendo encontrados 55 funcionários atuantes na SU. Verificou-se uma predominância de auxiliares de enfermagem $(60,0 \%)$, seguidos dos técnicos de enfermagem $(36,6 \%)$ e enfermeiros $(3,4 \%)$. Principais resultados: diversidade de condutas frente ao procedimento de limpeza e desinfecção dos materiais; ausência de supervisão direta do enfermeiro na SU, de manual de normas e rotinas, de programa de educação permanente. Tais resultados evidenciam a necessidade de recomendações de estratégias direcionadas à prevenção e controle de riscos referente às práticas de preparo dos materiais na instituição.

\section{ABSTRACT}

The aim of this study was to describe the profile of the professionals actives in the room of utilities of a university hospital concerning type of professional, supervision, availability of routines in the settings, inservice training and preparation of medical devices. This was a study of descriptive and quantitative approach undertaken in the period October 2006 to February 2007. For conducted this study it was used semi structured questionnaire. During the study, eleven units were assessed and found 55 employees working in SU. There was a predominance of assistant nursing (60.0\%), followed by technicians of nursing (36.6\%) and two nurses (3.4\%). The following results out standings: multiplicity conducts front of the procedure for cleaning and disinfecting medical devices, lack of direct supervision of the nurse in the SU, of a manual of rules and routines, of a program of continuing education. It was noted that these results highlight the need for recommendations for strategies directed to the prevention and control of risks relating to the practices of preparation of medical devices in the institution of study.

\section{INTRODUCCIÓN}

El reprocesamiento de equipamientos médicos de los hospitales consiste en una secuencia de actividades que comienza cuando el artículo es desmontado, seguido por la limpieza, inspección, embalado, etiquetado y la esterilización para garantizar el rendimiento y la seguridad del mismo ${ }^{(1)}$.

Entre las etapas de reprocesamiento, la limpieza es el momento de la eliminación de la suciedad, previniendo que la materia orgánica ponga en peligro el contacto directo entre el agente de desinfección o esterilización y el equipo, se constituye así en una etapa esencial para garantizar la calidad de la esterilización. El proceso de limpieza, y cuando sea necesario desinfección, previo a la esterilización es realizado generalmente en las salas de depósito de instrumental y material residual (SDI) de los establecimientos de cuidado de la salud (lugar para la recepción, limpieza, desinfección y separación de cualquier material contaminado, así como el envasado temporal de residuos) ${ }^{(2)}$

Así, los procedimientos realizados en las salas de depósito de instrumental y material residual exigen una permanente capacitación del equipo más allá de la adecuada supervisión de la enfermera profesional.

Sin embargo, la mayor parte del tiempo, esas salas no siempre reciben la debida atención, ya sea en términos de educación continua del personal que desarrolla las actividades de limpieza y preparación de material para ser enviado al centro de esterilización, o en 
infraestructura, a menudo con insuficiente dimensionamiento del área de trabajo, sin una adecuada ventilación e iluminación, y la escasez de insumos y equipos para la limpieza del material.

En consecuencia, las actividades de limpieza y preparación del material cuando se realizan incorrectamente pueden presentar riesgos para el paciente y favorecen la aparición de complicaciones asociadas con los procedimientos realizados, como la infección cruzada, pirógenas y reacciones tóxicos derivadas de los residuos de productos utilizados para la limpieza, desinfección y/o esterilización ${ }^{(1,3)}$.

En este proceso, la enfermera es responsable de la planificación de las acciones y su evaluación continua, además de la supervisión y la capacitación de personal permanente, ya que el reprocesamiento de los artículos requiere conocimiento, habilidad y formación de los técnicos que participan en esta actividad para garantizar la calidad del servicio y seguridad para los pacientes y los profesionales ${ }^{(1,3)}$.

Por lo tanto, teniendo en cuenta la importancia y la complejidad de esta práctica y la escasez de publicaciones sobre el tema, el estudio tuvo como objetivo describir el perfil de los profesionales que trabajan en la sala depósito de instrumental y material residual de un hospital universitario en lo relacionado a su formación, supervisión, la disponibilidad de rutinas en la unidad, el entrenamiento en el trabajo y la preparación de materiales.

\section{MATERIALES Y MÉTODOS}

Se trata de un estudio descriptivo y de enfoque cuantitativo, desarrollado en el período de octubre de 2006 a febrero de 2007, en once salas de depósito de instrumental y material residual de un hospital universitario, el cual presta atención terciaria, de gran tamaño y de referencia para el sistema de salud municipal y estatal en la asistencia sanitaria a los pacientes con enfermedades de media y alta complejidad. La clientela de la institución está constituida en el 95\% por pacientes provenientes del Sistema Unificado de Salud (SUS) y $5 \%$ de planes privados y/o particulares.

Los sectores del hospital objetos de investigación fueron clasificados como unidades de apoyo: para el diagnóstico y propedéutica; unidades de internamiento: para el alojamiento y la atención de los pacientes, por un período igual o mayor a 24 horas, y centro quirúrgico: unidad para el desarrollo de actividades quirúrgicas, así como la recuperación postanestesia y el post-operatoria inmediata (2).

Para la recolección de datos, se utilizó la técnica de entrevista estructurada mediante un cuestionario en el que se abordan cuestiones relacionadas con la categoría profesional, antigüedad en la SDI, la última vez que recibieron capacitación en la institución, si existe supervisión, la presencia de rutinas y la conducción del proceso de limpieza y desinfección de los equipamientos médico-hospitalarios en la SDI.

Las entrevistas fueron llevadas a cabo por dos ayudantes de investigación, estudiantes de licenciatura en Enfermería, previamente capacitados y supervisados por dos profesores de la Escuela de Enfermería.

La recolección de datos se produjo después de la aprobación por el Comité de Ética e Investigación registrado con № 267/033, teniendo en cuenta la resolución 196/96 de la Comisión Nacional de Ética de la Investigación, la que establece las normas que regulan la investigación con seres humanos. 
El encuentro con los profesionales fue en el lugar de trabajo de los mismos, y antes de la entrevista se les explicó los propósitos y objetivos del estudio, garantizando el mantenimiento del secreto, la confidencialidad y la preservación de sus identidades. Los participantes del estudio fueron todos los profesionales destacados a las SDI y que estaban presentes en los días que se realizaron las entrevistas, con excepción de los que se encontraban de vacaciones $\mathrm{y} / \mathrm{o}$ con licencias.

Para el análisis, los datos fueron codificados y digitados utilizando el software SPSS (Statistical products and Service Solutions), versión 13,0. En el análisis de los datos se utilizó la estadística descriptiva para caracterizar la muestra en estudio, siendo los datos presentados en forma de cuadros con frecuencias absoluta y relativa.

\section{RESULTADOS Y DISCUSIÓN}

Durante el estudio se contactó con 55 trabajadores de las SDIs. De acuerdo con la categoría profesional, hubo un predominio de auxiliares de enfermería $(60,0 \%)$, seguido de técnicos de enfermería $(36,6 \%)$ y llama la atención la presencia de enfermeras $(3,4 \%)$, actuando en esa actividad en dos áreas de apoyo.

El profesional de enfermería, teniendo en cuenta su competencia técnica puede realizar tales actividades, pero tiene como responsabilidad específica planificar, organizar y administrar la unidad para la atención, incluida la educación y la supervisión permanente del personal, además de sistematizar la atención. De ello se desprende que la presencia de profesionales de enfermería en la actividad de preparación de materiales se debe a la falta de empleados específicos para ese tipo de actividad que, en teoría, debe realizarse sólo por auxiliares y técnicos de enfermería.

En la Tabla 1 se muestran los resultados para las variables: presencia de supervisión directa de la enfermera, entrenamiento y la presencia de rutinas en las salas de depósito de instrumental y material residual.

Tabla 1 - Distribución de las variables: presencia de la supervisión directa de la enfermera, entrenamiento y presencia de rutinas en las salas de depósito de instrumental y material residual. Belo Horizonte, 2007.

\begin{tabular}{lllll}
\hline \multicolumn{1}{c}{ Variable } & Si & $(\%)$ & No & $(\%)$ \\
\hline Supervisión de la enfermera & 39 & $(73,6)$ & 14 & $(26,4)$ \\
Recibió entrenamiento & 17 & $(30,9)$ & 38 & $(69,1)$ \\
Existe manual de normas y rutinas & 38 & $(69,1)$ & 17 & $(30,9)$ \\
\hline
\end{tabular}

De conformidad con la Tabla 1, la mayor parte de los funcionarios recibieron supervisión directa de las enfermeras, sin embargo, esta no se produjo de manera constante y sistemática.

Se hace hincapié en que la supervisión directa de la enfermera favorece la seguridad y la calidad para la preparación de materiales a ser reprocesados. Además, este profesional contribuye a minimizar la ocurrencia de eventos adversos derivados de la limpieza y desinfección.

Sin embargo, cuando se evaluó la forma de supervisión de las enfermeras entre los profesionales que dicen recibir la mayor parte de la supervisión (66\%) esta acontece de acuerdo como es demandado (Figura 1). 
Figura 1. Distribución de los profesionales entrevistados en las salas de depósito de instrumental y material residual, que informaron tener supervisión de la enfermera en sus actividades. Belo Horizonte, 2007.

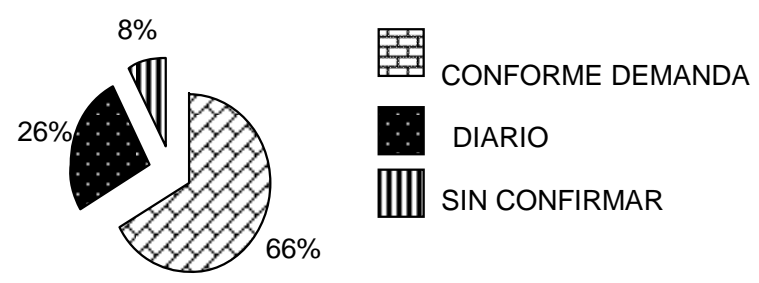

Esta observación nos lleva a preguntarnos sobre las condiciones en las que los funcionarios están ejecutando las actividades en las salas de depósito de instrumental y material residual, teniendo en cuenta los riesgos inherentes en los procedimientos de limpieza y desinfección de materiales, además de los riesgos laborales a que están expuestos con frecuencia, inherente a ese tipo de actividad, como la ocurrencia de accidentes relacionados con material biológico ${ }^{(4)}$.

En cuanto a la experiencia en el puesto del personal en las salas de depósito de instrumental y material residual, se constató que la mayoría de los funcionarios tenían un tiempo de más de un año, siendo más frecuente el rango de uno a cinco años y más de diez años, y no hubo registro en el rango de cinco a diez años (Gráfico 2).

Gráfico 2 - Distribución de los funcionarios que actúan en las salas de depósito de instrumental y material residual de acuerdo con tiempo de experiencia en las diferentes unidades. Belo Horizonte, 2007.

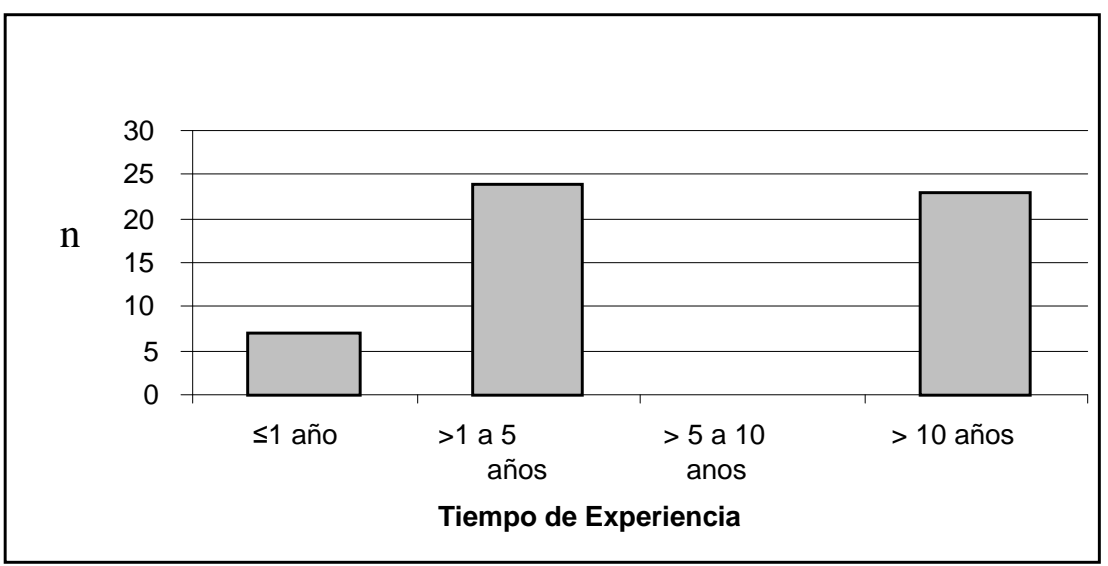

En cuanto a la capacitación específica en reprocesamiento de materiales, la mayoría de los empleados $(69,1 \%)$ dijeron que no habían recibido entrenamiento sobre cómo preparar las materias en las salas de depósito de instrumental y material residual. Sin embargo, cuando se le preguntó la última vez que recibieron entrenamiento, para la mayoría de los profesionales se produjo entre hace más de un año y menos de cinco años (Gráfico 3). 
Gráfico 3. Distribución de los profesionales entrevistados de las salas de depósito de instrumental y material residual, según su último entrenamiento. Belo Horizonte, 2007.

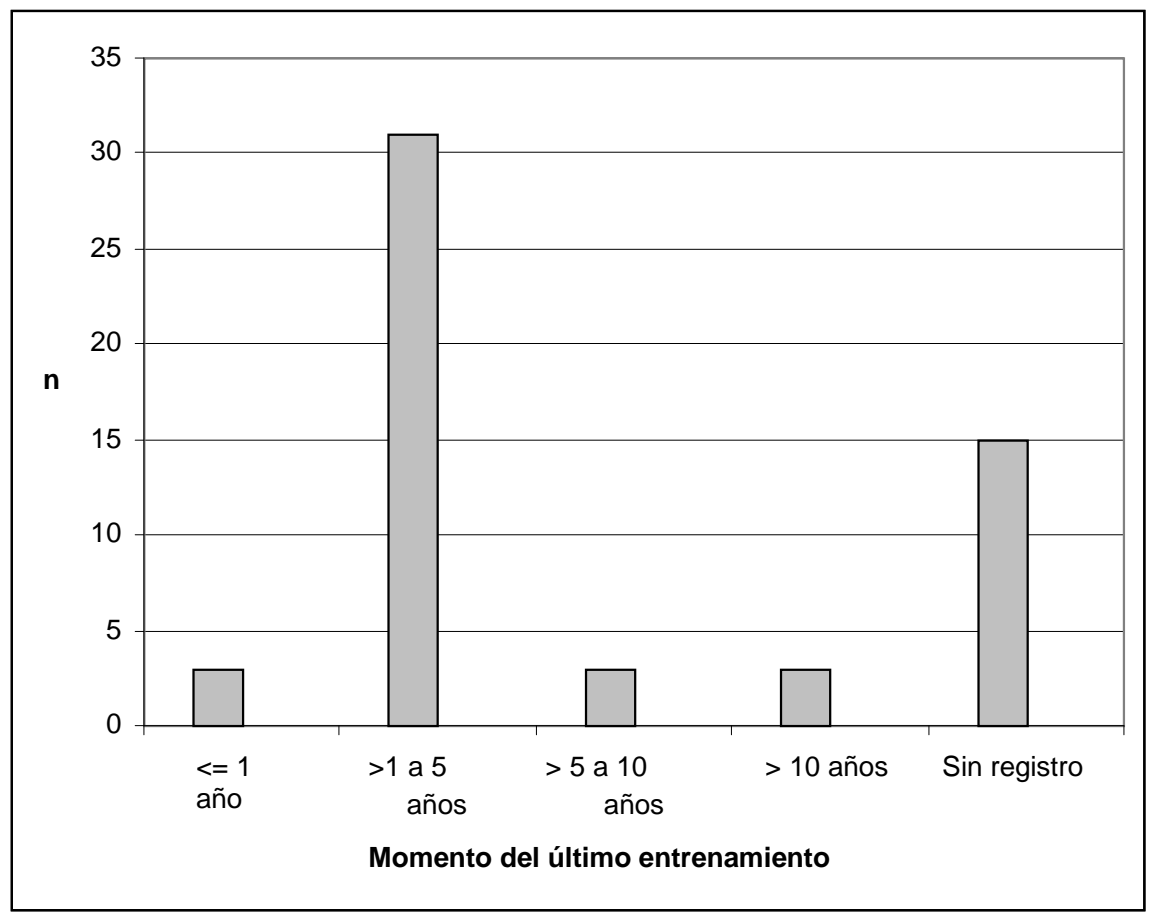

De este modo, se puede inferir que los empleados que tienen una antigüedad en el servicio de menos de 1 año y más de 10, probablemente no han recibido entrenamiento específico en relación con el reprocesamiento de materiales, lo cual plantea grandes interrogantes sobre la forma como realizan esa actividad.

Desarrollar un programa de entrenamiento es un aspecto clave para comprender la importancia de planificar las fases iniciales del reprocesamiento (limpieza, inspección y desinfección), y para el desarrollo de la competencia y habilidad de profesionales para cada una de estas tareas.

Cabe señalar que todos los funcionarios informaron de que fueron "acompañados por un colega" como medio de integración y entrenamiento en servicio durante el periodo de admisión, de una o dos semanas, y que este acompañamiento se restringió sólo a una observación práctica. Sin embargo, esta práctica debe ser vista con mucha atención porque, si bien puede ser útil para garantizar el mantenimiento de procedimientos estándar, también pueden incurrir en la repetición de los vicios que consolidan la aplicación de técnicas incorrectas e inseguras; y no es, de hecho, considerada como una manera adecuada la formación o inserción de nuevos empleados ${ }^{(4)}$.

Además, el aprendizaje enfocado en un abordaje repetitivo, donde sólo se destaca la habilidad práctica lleva a la formación de profesionales que simplemente ejecutan las tareas, disociando la reflexión crítica de la habilidad técnica, o sea, no hay una asociación entre la teoría y la práctica que favorecería la asimilación de un razonamiento lógico, reflexivo y consciente sobre la necesidad y la importancia del cuidado de los materiales y su riesgo potencial ${ }^{(5)}$.

Otro aspecto de suma importancia es la existencia de rutinas escritas en las unidades, lo que permite a los funcionarios adoptar conductas normalizadas para realizar las etapas de los procedimientos de limpieza, inspección y desinfección, teniendo en cuenta cada tipo de material y el grado de riesgo. 
En este estudio, los datos mostraron que la mayoría de los empleados $(69,1 \%)$ confirmó la existencia de rutinas escritas en la unidad. Sin embargo, el número de trabajadores que alegaron no contar con ese instrumento en la unidad en que trabajaban también fue significativo $(30,9 \%)$. La falta de rutinas hace que sea difícil la normalización de las actividades, la resolución de dudas, además de la integración y la adaptación de los nuevos empleados, lo que interfiere directamente en la calidad de su servicio durante el reprocesamiento de materiales médicos hospitalarios.

Sobre la base de estos datos, a los encuestados también se les preguntó acerca de los productos utilizados para la limpieza y desinfección de cada uno de los artículos que reprocesan en las salas de depósito de instrumental y material residual, y las respuestas están dadas en la Tabla 2.

Tabla 2 - Distribución del método utilizado por los funcionarios para llevar a cabo la limpieza y desinfección de materiales. Belo Horizonte, 2007.

\section{Sectores}

\begin{tabular}{|c|c|c|c|c|c|c|c|c|c|c|c|c|}
\hline & \multicolumn{4}{|c|}{ Apoyo } & \multicolumn{4}{|c|}{ Internación } & \multicolumn{4}{|c|}{ Centro quirúrgico } \\
\hline Materiales Limpieza & $\begin{array}{l}\text { AS } \\
(1)\end{array}$ & $\begin{array}{l}\mathbf{D} \\
\mathbf{E} \\
(2)\end{array}$ & $\begin{array}{l}A \\
S \\
+ \\
D \\
E\end{array}$ & $\begin{array}{l}\text { No } \\
\text { limpia }\end{array}$ & $\begin{array}{l}A \\
S\end{array}$ & $\begin{array}{l}\text { D } \\
\text { E }\end{array}$ & $\begin{array}{l}A \\
S \\
+ \\
D \\
E\end{array}$ & $\begin{array}{l}\text { No } \\
\text { limpia }\end{array}$ & $\begin{array}{l}A \\
S\end{array}$ & $\begin{array}{l}\text { D } \\
\text { E }\end{array}$ & $\begin{array}{l}\mathrm{A} \\
\mathrm{S} \\
+ \\
\mathrm{D} \\
\mathrm{E}\end{array}$ & $\begin{array}{l}\text { No } \\
\text { limpia }\end{array}$ \\
\hline \multicolumn{13}{|l|}{ Artículos } \\
\hline Bandeja de baño & 13 & $\begin{array}{l}0 \\
3\end{array}$ & 00 & 00 & 21 & 02 & 00 & 00 & 04 & 02 & 00 & 00 \\
\hline $\begin{array}{l}\text { Manguera de } \\
\text { Oxígeno }\end{array}$ & 18 & $\begin{array}{l}0 \\
3\end{array}$ & 05 & 00 & 18 & 02 & 03 & 00 & 00 & 02 & 01 & 00 \\
\hline Bacín (chata) & 19 & $\begin{array}{l}0 \\
3\end{array}$ & 00 & 00 & 21 & 02 & 00 & 00 & 04 & 02 & 00 & 00 \\
\hline $\begin{array}{l}\text { Cubo de } \\
\text { medicación }\end{array}$ & 18 & $\begin{array}{l}0 \\
3\end{array}$ & 05 & 00 & 21 & 02 & 00 & 00 & 04 & 02 & 00 & 00 \\
\hline Especulo vaginal & 04 & $\begin{array}{l}0 \\
3\end{array}$ & 00 & 01 & 04 & 01 & 00 & 00 & 01 & 02 & 00 & 00 \\
\hline Jarro para baño & 11 & $\begin{array}{l}0 \\
3\end{array}$ & 00 & 00 & 21 & 02 & 00 & 00 & 04 & 02 & 00 & 00 \\
\hline Pato (orinal) & 19 & $\begin{array}{l}0 \\
3\end{array}$ & 00 & 00 & 21 & 02 & 00 & 00 & 04 & 02 & 00 & 00 \\
\hline $\begin{array}{l}\text { Pinza } \\
\text { curativos }\end{array}$ & 10 & $\begin{array}{l}0 \\
3\end{array}$ & 13 & 00 & 21 & 02 & 00 & 00 & 00 & 06 & 00 & 00 \\
\hline $\begin{array}{l}\text { Tijera } \\
\text { curativos }\end{array}$ & 14 & $\begin{array}{l}0 \\
3\end{array}$ & 07 & 00 & 21 & 02 & 00 & 00 & 00 & 06 & 00 & 00 \\
\hline $\begin{array}{l}\text { Vidrio } \\
\text { aspiración }\end{array}$ & 18 & $\begin{array}{l}0 \\
0\end{array}$ & 02 & 02 & 20 & 02 & 01 & 00 & 00 & 01 & 00 & 00 \\
\hline Materitates Desinfección & $\underset{(3)}{A L}$ & $\begin{array}{l}\mathbf{H} \\
\mathbf{S} \\
(4)\end{array}$ & $\underset{(5)}{G}$ & $\begin{array}{l}\text { No } \\
\text { desinf } \\
\text { ecta }\end{array}$ & AL & $\begin{array}{l}\mathrm{H} \\
\mathrm{S}\end{array}$ & $\mathbf{G}$ & $\begin{array}{l}\text { No } \\
\text { desinfe } \\
\text { cta }\end{array}$ & $A L$ & $\begin{array}{l}H \\
S\end{array}$ & $\mathbf{G}$ & $\begin{array}{l}\text { No } \\
\text { desinf } \\
\text { ecta }\end{array}$ \\
\hline
\end{tabular}




\begin{tabular}{|c|c|c|c|c|c|c|c|c|c|c|c|c|}
\hline \multicolumn{13}{|l|}{ Artículos } \\
\hline Bandeja de baño & 08 & $\begin{array}{l}0 \\
0\end{array}$ & 01 & 06 & 08 & 01 & 00 & 00 & 00 & 00 & 00 & 06 \\
\hline $\begin{array}{l}\text { Manguera de } \\
\text { Oxígeno }\end{array}$ & 05 & $\begin{array}{l}0 \\
8\end{array}$ & 00 & 13 & 01 & 02 & 00 & 20 & 00 & 00 & 00 & 03 \\
\hline Bacín (chata) & 15 & $\begin{array}{l}0 \\
1\end{array}$ & 01 & 02 & 07 & 01 & 00 & 01 & 00 & 00 & 00 & 06 \\
\hline $\begin{array}{l}\text { Cubo } \\
\text { medicación }\end{array}$ & 05 & $\begin{array}{l}0 \\
0\end{array}$ & 00 & 21 & 06 & 00 & 00 & 16 & 00 & 00 & 00 & 06 \\
\hline Especulo vaginal & 02 & $\begin{array}{l}0 \\
1\end{array}$ & 00 & 05 & 00 & 00 & 00 & 04 & 00 & 00 & 00 & 03 \\
\hline Jarro para baño & 03 & $\begin{array}{l}0 \\
0\end{array}$ & 00 & 09 & 08 & 01 & 00 & 00 & 00 & 00 & 00 & 06 \\
\hline Pato (orinal) & 14 & $\begin{array}{l}0 \\
1\end{array}$ & 01 & 02 & 07 & 01 & 00 & 01 & 00 & 00 & 00 & 06 \\
\hline $\begin{array}{l}\text { Pinza } \\
\text { curativos }\end{array}$ & 03 & $\begin{array}{l}0 \\
9\end{array}$ & 09 & 13 & 03 & 00 & 00 & 20 & 00 & 00 & 00 & 06 \\
\hline $\begin{array}{l}\text { Tijera } \\
\text { curativos }\end{array}$ & 03 & $\begin{array}{l}0 \\
0\end{array}$ & 00 & 20 & 03 & 00 & 00 & 20 & 00 & 00 & 00 & 06 \\
\hline $\begin{array}{l}\text { Vidrio } \\
\text { aspiración }\end{array}$ & 04 & $\begin{array}{l}1 \\
0\end{array}$ & 00 & 05 & 04 & 02 & 00 & 05 & 00 & 00 & 00 & 01 \\
\hline
\end{tabular}

De conformidad con la Tabla 2, existe una diversidad de conductas relacionadas con los productos de limpieza y desinfección utilizados por los funcionarios de las salas de depósito de instrumental y material residual, para el mismo material o artículo. Es importante destacar que, a menudo, esa diferencia se produjo incluso entre los funcionarios de la misma unidad, en diferentes turnos de trabajo, lo cual puede ser una consecuencia directa de la falta de un programa de educación continua y de la ausencia de un manual de rutinas.

Otra observación que llamó la atención fue la dificultad de los funcionarios para distinguir el jabón líquido del detergente enzimático, lo que demuestra el desconocimiento de la finalidad del uso de cada uno de estos productos, así como de su correcta indicación.

La existencia de comportamientos no estandarizados en la institución, en relación con la limpieza y desinfección de materiales, en la que el propio funcionario instituyó la rutina de trabajo sin fundamentación para esa práctica, su seguridad y su idoneidad, es uno de los factores que pueden contribuir al surgimiento de eventos adversos, principalmente las infecciones (presencia de biofilms y endotoxinas) e intoxicación debido a la presencia de residuos de productos químicos utilizados para la limpieza y la esterilización.

El proceso de limpieza llevado a cabo con agua y detergente enzimático permite la eliminación de suciedad adherida a las superficies, grietas, sierras y las articulaciones del material médico hospitalario. Por lo tanto, el detergente enzimático actúa como degradante de las proteínas de la sangre y los fluidos corporales por acción de la proteasa, lipasas y amilasas presentes en los mismos, fomentando la limpieza química en los lugares de difícil acceso, como lúmenes largos y estrechos, por ejemplo las mangueras de oxígeno, de aspiración y de los endoscopios ${ }^{(6)}$. 
Así, la limpieza con detergente enzimático permite una mayor reducción de carga microbiana viable (reducción de hasta $10^{5}$ de carga bacteriana) y del biofilm, conjunto de células microbianas, componentes sanguíneos y materiales no celulares que permanecen adheridos a las superficies de los materiales ${ }^{(7-8)}$.

Se destaca también el hecho de que algunos funcionarios no realizan la limpieza de determinados materiales en la unidad, ya que la Central de Material Esterilizado (CME) del hospital de estudio está clasificada como semi-centralizada (el material debe limpiarse en las unidades y luego encaminado a la CME para ser esterilizado) ${ }^{(9)}$.

En el contexto de los procedimientos de desinfección (proceso de eliminación de todos los microorganismos en la forma vegetativa, independientemente de su clasificación en cuanto a la patogenicidad) en la literatura se indica el etanol ( $A L)$, al $70 \%$ por su actividad germicida, su menor costo y menor toxicidad. Su mecanismo de acción es la desnaturalización de proteínas. Se recomienda para desinfección de nivel medio de artículos un tiempo de exposición de diez minutos, con tres aplicaciones intercaladas con secado natural ${ }^{(10-11)}$.

El hipoclorito de sodio (HS) se recomienda para la desinfección y descontaminación de las superficies, mas es difícil determinar su validez por la posibilidad de perder su estabilidad. Presenta un olor característico, toxicidad, además de ser fácilmente inactivado en presencia de materia orgánica es corrosivo para metales ${ }^{(10-11)}$.

Y, por último, el glutaraldehído (G) recomendado para los artículos semi-críticos e instrumentos sensibles al calor se utiliza para la desinfección en la concentración del $2 \%$ por un período de exposición de 20 a 30 minutos. No ocasiona daños a productos de caucho, metales, lentes y otros materiales, pero su uso ha sido replanteado por el riesgo de impregnación en materiales porosos, además de los riesgos laborales a los funcionarios, por lo cual, cuando es usado, debería considerarse la importancia de un entorno con circulación de aire, ventilado y usando Equipos de Protección Individual (EPI) por el operador, además de la necesidad de un riguroso enjuague del material para reducir la posibilidad de su impregnación ${ }^{(10-11)}$.

Como se ve, el glutaraldehído, debido a los riesgos de toxicidad y de impregnación, no debería utilizarse. Los efectos secundarios relacionados con el uso de este producto han sido citados por los propios funcionarios durante la recolección de datos, tales como alergias y síntomas respiratorios, especialmente la dificultad de manejo del mismo por falta de infraestructura, medio ambiente aireado y ventilado.

Asimismo, no obstante las recomendaciones para la desinfección de material médico hospitalario, se constató que los profesionales entrevistados no fueron capacitados para realizar estas técnicas y la diferenciación de las mismas según la clase de materiales y su grado de contaminación.

\section{CONSIDERACIONES FINALES}

En el análisis general de los resultados obtenidos, se comprueba que las revelaciones del estudio son muy importantes, mostrando la necesidad de recomendaciones para el establecimiento de estrategias dirigidas a la prevención y el control de los riesgos relativos a las prácticas de preparación de materiales.

De acuerdo con los datos presentados, hay una necesidad de vigilar el proceso de limpieza y principalmente desinfección de las salas de depósito de instrumental y material residual, 
frente a los diferentes comportamientos y el uso de los productos descritos por los funcionarios. Por otra parte, también merece atención la implementación de rutinas y protocolos con indicaciones de productos desinfectantes, las indicaciones recomendadas por el fabricante, acción, tiempo de exposición, enjuague y forma de embalaje de los materiales después de la desinfección. Especialmente llamó la atención lo siguiente:

a) la falta de funcionarios específicos para las salas de depósito de instrumental y material residual, siendo en algunas unidades tal actividad ejercida por la enfermera profesional;

b) la ausencia de supervisión directa por personal de enfermería, a pesar de que los funcionarios manifestaron que existe; se constató que la supervisión no siempre actúa con la frecuencia demandada;

c) la diversidad de conductas frente al procedimiento de limpieza y desinfección de los materiales utilizados por los funcionarios de las salas de depósito de instrumental y material residual y hasta de la misma unidad, en diferentes turnos de trabajo;

d) el acceso todos los funcionarios de la SDI a un manual de normas y a rutinas normalizadas y actualizadas;

e) ausencia de un programa de educación continua, incluido el entrenamiento periódico y la supervisión de los empleados en esas actividades.

\section{Las estrategias recomendadas:}

a) reorganización de las salas de depósito de instrumental y material residual con la normalización y actualización de las normas y rutinas, facilitando el acceso a ese instrumento para consulta y orientación de todos los empleados, lo que evitaría la diversidad de conductas e imposición de rutinas propias sin fundamentación de esa práctica, de su seguridad y conveniencia;

b) implementación de un programa de supervisión de la enfermera a los funcionarios de las salas de depósito de instrumental y material residual de una forma más directa y presente;

c) la implementación de un programa de educación continua, incluido el entrenamiento periódico y la supervisión de los empleados en esas actividades, donde se debe acentuar la responsabilidad del trabajo realizado y el riesgo inherente en los procedimientos de manipulación, desinfección, esterilización y almacenamiento de materiales, buscando así la seguridad del paciente y de los propios trabajadores.

A pesar de la importancia del tema en cuestión, cabe destacar, como una limitación y al mismo tiempo un desafío, la escasez de referencias a la evaluación de las prácticas en las salas de depósito de instrumental y material residual que impliquen el reprocesamiento de artículos, tanto en Brasil como en el resto del mundo.

De este modo, estas dificultades también se reflejan en la construcción de la revisión de la literatura y en el análisis y discusión de los resultados. Por último, se espera que este estudio contribuya al avance de los conocimientos en este ámbito, con el fin de desvelar la práctica de esos profesionales y fomentar nuevos estudios.

\section{REFERÊNCIAS BIBLIOGRÁFICAS}

1) Brasil. Resolución $n^{\circ} 2606$ del 11 de agosto de 2006. Dispone sobre las directrices para elaboración, validación e implantación de protocolos de reprocesamiento de productos médicos y da otras providencias. Diário Oficial da União, Brasília, 14 ago. 2006. 
2) Brasil. Ministério da Saúde. Resolución RDC 50 del 21 de febrero de 2002. Dispone sobre el Reglamento Técnico para planeamiento, programación, elaboración y evaluación de proyectos físicos de estabelecimientos asistenciales de salud. Diário Oficial da União, Brasília, 21 feb. 2002 [acceso en 20 dic 2006]. Disponible en: <www.anvisa.gov.br>.

3) Dumartin C, Brücker G. Rules of decontamination and disinfection of medicosurgical instruments in the operating room. Ann Chir. 1995; 49(2):173-9.

4) Tipple AFV, Souza TR, Bezerra ALQ, Munari DB. O trabalhador sem formação em enfermagem atuando em centro de material e esterilização: desafio para o enfermeiro. Rev Esc Enferm USP. 2005; 39(2):173-80.

5) Souza MCB, Ceribelli MIPF. Enfermagem no centro de material esterilizado- A prática da educação continuada. Revista Latino-americana de Enfermagem. 2004;12(5):767-74.

6) Fernandes AT, Fernandes MOV, Filho NR. Infecção Hospitalar e suas interfaces na área da saúde. São Paulo: Editora Atheneu; 2000.

7) Silva MV. Influência da Reutilização na Biocompatibilidade de Materiais Médicohospitalares de Uso Único [tesis]. São Paulo: Universidad de São Paulo, 2002.

8) Ribeiro SPC. Reprocessamento de cateteres de angiografia cardiovascular após uso clínico e contaminados artificialmente: avaliação da eficácia da limpeza e esterilização [tesis]. São Paulo: Escuela de Enfermería, Universidad de São Paulo; 2006.

9) Mussel IC, Armond GA, Oliveira AC. Central de material esterilizado. In: Oliveira AC, MEDSI/Guanabara Koogan. Infecção Hospitalar, epidemiologia, prevenção e controle. Rio de Janeiro: 2005. p. 531-42.

10) Graziano KU. Processos de Limpeza, Desinfecção e Esterilização de artigos médicohospitalares. In: Oliveira AC, MEDSI/Guanabara Koogan. Infecção Hospitalar, epidemiologia, prevenção e controle. Rio de Janeiro: 2005. p. 491-516.

11) Brasil. Ministério da Saúde. Procesamiento de Artículos y Superficies en Estabelecimientos de Salud. Diário Oficial da União, Brasília, 1994. 


\section{Anexo 1 -}

Sector

Soporte: ( )

Internación: ( )

Centro

Quirúrgico:

Unidad:

1. PROFESIONAL:

\begin{tabular}{|c|c|}
\hline 1. Capacitación y Entrenamiento & Momento del último entrenamiento \\
\hline $\begin{array}{l}\text { Recibió entrenamiento específico en } \\
\text { tratamiento de materiales (limpieza, } \\
\text { desinfección y esterilización) antes de } \\
\text { asumir las actividades en las salas de } \\
\text { depósito de instrumental y material } \\
\text { residual? } \\
\text { Sí () No () }\end{array}$ & $\begin{array}{l}<1 \text { año } \\
>1 \text { año a } 5 \text { años } \\
>5 \text { a } 10 \text { años } \\
>10 \text { años }\end{array}$ \\
\hline 1.2. Supervisión & $\begin{array}{l}\text { ¿Con qué frecuencia se produce } \\
\text { supervisión? }\end{array}$ \\
\hline $\begin{array}{l}\text { Sus actividades en las salas de } \\
\text { depósito de instrumental y material } \\
\text { residual son supervisadas } \\
\text { directamente por la enfermera? } \\
\text { Sí ( ) No ( ) }\end{array}$ & $\begin{array}{l}\text { Diario } \\
\text { Semanal } \\
\text { Mensual } \\
\text { No sabe indicar } \\
\text { Como la demanda }\end{array}$ \\
\hline
\end{tabular}

2. MATERIALES

\begin{tabular}{|l|l|l|l|l|l|l|l|}
\hline \multirow{2}{*}{ Material } & \multirow{2}{*}{ Limpieza } & \multicolumn{5}{|l|}{ Desinfección } & \multirow{2}{*}{ CME } \\
\cline { 4 - 7 } & & \multicolumn{2}{|c|}{ Química } & Física & \\
\hline & AS & DE & HS & AL & G & Estufa & \\
\hline Pinza para curativo & & & & & & & \\
\hline Tijeras para curativos & & & & & & & \\
\hline Balde para baño & & & & & & & \\
\hline Manguera de oxígeno & & & & & & & \\
\hline Bacín (chata) & & & & & & & \\
\hline Pato (orinal) & & & & & & & \\
\hline Jarro para baño & & & & & & & \\
\hline Cubo de medicación & & & & & & & \\
\hline Vidrio de aspiración & & & & & & & \\
\hline Especulo vaginal & & & & & & & \\
\hline
\end{tabular}

Legenda:

AS - agua y jabón

DE - detergente

enzimático

HS- hipoclorito de sodio

AL-alcohol

G- glutaraldeído

CME- Central de

Material

Esterilizado

\section{RUTINAS:}

Hay manual de normas y rutinas escritas en su unidad relacionadas con el reprocesamiento de

los

Sí ( ) No

artículos

y

equipamientos?

Fecha

Responsable (recolección): 